\title{
Optimizing patient centered care in the cardiac intensive care unit: Harness the safety, effectiveness, and incremental value of radionuclide perfusion, function, and molecular imaging
}

\author{
Peter A. Bleszynski, MD, ${ }^{\text {a }}$ and Ronald G. Schwartz, MD, MS, FACC, FAHA, \\ ABNM, MASNC ${ }^{\mathrm{a}, \mathrm{b}}$ \\ a Cardiology Division, Department of Medicine, University of Rochester Medical Center, \\ Rochester, NY \\ b Nuclear Medicine Division, Department of Imaging Sciences, University of Rochester Medical \\ Center, Rochester, NY
}

Received May 25, 2021; accepted May 26, 2021

doi: 10.1007/s12350-021-02691-x

\section{See related article, pp. 553-569}

Cardiovascular disease (CVD) and its sequalae remain the number one cause of death in the USA and globally. Despite a heightened focus on cardiac risk stratification, primary and secondary prevention, and an increasing number of medical and mechanical therapies, CVD and its management have grown in scope and complexity. Nuclear cardiology techniques provide safe and effective evaluation of CVD. Safety is our professional and ethical imperative, and in critically ill patients with cardiovascular, CNS, pulmonary, renal and/or hepatic dysfunction, nuclear cardiology techniques reliably provide safe assessment of diagnosis, prognosis, and treatment response. Predischarge vasodilator stress testing and its safe, prompt, and effective reversal with either aminophylline, theophylline or caffeine provide an exemplary standard of safety and effectiveness and wide applicability to patients in the field of cardiovascular stress testing. ${ }^{1,2}$ Beyond safety, the quantitative assessment of myocardial relative perfusion, absolute

Reprint requests: Ronald G. Schwartz, MD, MS, FACC, FAHA, ABNM, MASNC, Nuclear Medicine Division, Department of Imaging Sciences, University of Rochester Medical Center, Rochester, NY ; ronald_schwartz@urmc.rochester.edu J Nucl Cardiol 2023;30:570-3.

$1071-3581 / \$ 34.00$

Copyright (C) 2021 American Society of Nuclear Cardiology. myocardial blood flow and blood flow reserve, global and regional function, dyssynchrony, ventricular volumes and molecular imaging of sympathetic innervation, dysrhythmia, infiltrative cardiomyopathies and aortopathies, prosthetic valve endocarditis, and cardiac device infection endow the discipline of nuclear cardiology with the signature value of incremental assessment of diagnosis, prognosis, and treatment response in a wide range of cardiac conditions.

In the current issue of the Journal, Schenone et al. provide an overview of the safety and utility of radionuclide imaging of patients in the coronary intensive care unit (CICU). ${ }^{3}$ The authors note that the evaluation of CICU patients is guided by several considerations: (1) Is the current presentation driven by myocardial ischemia or solely represent a progression of cardiomyopathy?, (2) Do benefits of revascularization outweigh the risks on the basis of myocardium at risk?, and (3) Which vessels/territories would benefit from revascularization? A fourth consideration in their review is the influence of other disease processes that may contribute to the presentation of the acutely ill patient in the CICU. Major take-home points of this Cleveland Clinic review merit further consideration. The versatility of nuclear cardiology applications in CICU is summarized in the Figure. ${ }^{3}$ 


\section{Utility of Cardiovascular Nuclear Imaging in the Cardiac Intensive Care Unit}
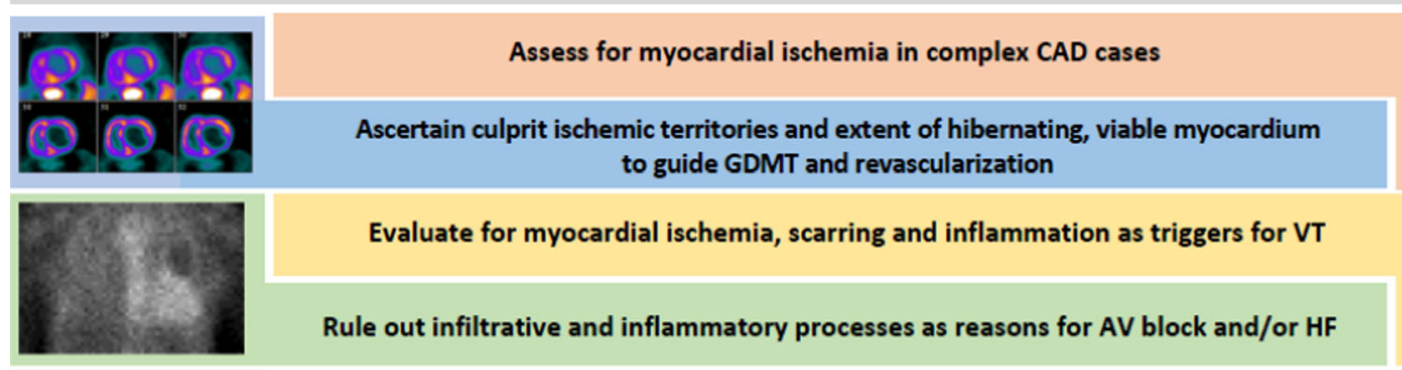

Evaluate for myocardial ischemia, scarring and inflammation as triggers for VT

Rule out infiltrative and inflammatory processes as reasons for AV block and/or HF

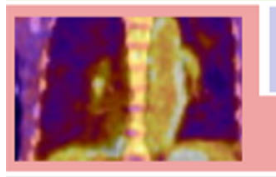

Assist in the diagnosis of Infective endocarditis in complex cases

Discriminate inflammatory aortopathies from acute aortic syndrome in selected cases

Advantages of Nuclear Imaging in the Cardiac Intensive Care Unit

- Minimal patient cooperation required.

- Relatively short imaging acquisition times.

- Image acquisition not precluded by cardiac devices such as PPM/ICD, or IABP.

- Imaging not precluded by abnormal liver or renal function.

\section{ASSESS FOR MYOCARDIAL ISCHEMIA IN COMPLEX CAD CASES}

Global and culprit regional myocardial ischemic jeopardy can be readily quantified by PET and SPECT MPI. Extensive global LV stress-induced ischemia that exceeds the burden of infarction identifies near-term benefit of revascularization. ${ }^{4,5}$ Incremental value of revascularization has been clearly identified by PET measurements of MBF and MFR. ${ }^{6-9}$ Patients with $\geq$ $10 \%$ stress-induced ischemic defect and MFR $\leq 1.8$ on PET MPI have been reported to benefit from revascularization. ${ }^{9}$ Patients with stress $\mathrm{MBF}<1.8 \mathrm{ml} / \mathrm{min} / \mathrm{gr}$ and MFR $<2.0$ also benefit from revascularization. ${ }^{7} \mathrm{~A}$ growing literature supports the use of CZT SPECT to obtain MBF and MFR results that correlate highly with those of PET and coronary angiography. ${ }^{10,11}$

\section{ASCERTAIN CULPRIT ISCHEMIC TERRITORIES AND EXTENT OF HIBERNATING VIABLE MYOCARDIUM TO GUIDE GDMT AND REVASCULARIZATION}

A major question confronting management of patients who survive acute myocardial infarction with extensive cardiac dysfunction is whether sufficient ischemic jeopardy exists to provide benefit of revascularization. The Ottawa-FIVE sub-study of the PAAR2 trial is unique in the literature in providing a robust, meaningful study design to evaluate this question. This secondary analysis of this interventional trial evaluated the effect of revascularization on primary cardiovascular outcome events as a function of the extent of hibernating viable myocardium (HVM) defined by "mismatched" PET resting myocardial perfusion defects associated with increased myocardial FDG uptake representing myocardial glycolysis associated with regional ischemia. The study showed that revascularization was increasingly effective in reducing primary cardiovascular outcome events with progressive increases in the extent of HVM $\geq 7 \%$ LV myocardium. ${ }^{12}$ Similar to the diagnostic accuracy of PET perfusion metabolism mismatch, HVM can comparably be evaluated by restredistribution thallium-201 imaging. ${ }^{13}$ An opportunity exists to confirm with randomized controlled trials the threshold of HVM by either technique, including CZT SPECT ultra-low-dose thallium-201 rest-redistribution imaging. Nevertheless, compelling observational data have stimulated clinical application of assessment of HVM, rather than "viability" without evaluation of hibernation prior to revascularization by radionuclide PET and SPECT imaging of perfusion, cellular energy metabolism, and myocyte membrane function evidenced by cation redistribution. Importantly, future studies that attempt to quantify the benefit of revascularization 
should consider the substrate of global ischemic burden as the sum of stress-induced ischemia and resting ischemia in dysfunctional, viable myocardium that SPECT and PET myocardial perfusion, function, and metabolic imaging can identify.

\section{EVALUATE FOR MYOCARDIAL ISCHEMIA, SCARRING, AND INFLAMMATION AS TRIGGERS FOR VT}

CICU patients with monomorphic VT associated with predominant scar on SPECT or PET MPI suggest the value of antiarrhythmic therapy, VT ablation, and/or ICD implantation. CICU patients with polymorphic VT/VF with large areas of ischemia by SPECT of PET MPI are likely to benefit from ischemia-guided revascularization. Malignant polymorphic VT/VF in CICU patients with evidence of active inflammatory cardiac sarcoidosis (CS) can be refractory to standard anti-arrhythmic therapy or revascularization and benefit from intensive immunosuppression and implantation of a dual chamber ICD. These cases of malignant polymorphic VT/VF associated with active inflammatory myocardial sarcoidosis without scar may not be identified by cardiac MRI. The residual risk of VT and VF on immunosuppressive therapy can be uniquely identified by resolution of FDG uptake on serial CS PET imaging, thereby facilitating timely tapering and discontinuation of immunosuppressive therapies, limiting numerous adverse effects and enhancing quality of life associated with these lifesaving therapies.

\section{RULE OUT INFILTRATIVE AND INFLAMMATORY PROCESSES AS REASONS FOR AV BLOCK AND/ OR HF}

Intense, heterogenous FDG uptake on PET scan after prolonged (12-18 hour) fasting preceded by very lowcarbohydrate high-fat meals identifies active inflammatory CS in most cases in the presence or absence of scar in an atypical (non-vascular) distribution. ${ }^{14}$ Extensiveness of FDG uptake and RV uptake contribute to adverse prognosis. In addition to aforementioned polymorphic VT refractory to anti-dysrhythmic therapy, the classic presentations of active inflammatory sarcoidosis include high-degree AV block and HFrEF. HF may result from extensive CS infiltration, also suggestive of myocarditis, or from myocardial stunning associated with atrial and ventricular tachyarrhythmias. The presence of AV block or HF unexplained by myocardial infarction or ischemia in CICU patients suggest the value of fasting FDG PET in combination with resting PET or hybrid CZT SPECT MPI to diagnose active CS inflammation and scar, to initiate timely immunosuppressive therapy and limit progression of cardiac degeneration.

\section{ASSIST IN THE DIAGNOSIS OF INFECTIVE ENDOCARDITIS IN COMPLEX CASES}

Schenone et al. review the role of fasting FDG PET imaging to evaluate prosthetic valve endocarditis in cases where echocardiography is inconclusive or suggests aortic root abscess. ${ }^{15}$ Detection of IE has high sensitivity and limited specificity; and diagnostic accuracy can be optimized by follow-up testing with an indium-111 WBC scan. ${ }^{16}$ Most patients with a prosthetic valve and no evidence of infection exhibit prosthetic F18-FDG valve uptake 1 year after surgery, mostly with a diffuse homogeneous uptake and occasionally diffuse heterogenous or focal which may simulate $\mathrm{CS}^{.}{ }^{17} \mathrm{In}$ CICU patients, focal F18-FDG uptake can reflect other non-infectious conditions including thrombi, non-calcified atherosclerotic plaque, vasculitis, tumor, and foreign body reactions. The interpretation of an abnormal uptake on either F18-FDG PET/CT or WBC scans as a major criterion for the diagnosis of IE is supported by the European Society of Cardiology for the management of IE. ${ }^{18}$ Schenone et al. suggest that the diagnosis and management of endocarditis in complex patients utilize hybrid PET/CT imaging and a multi-disciplinary endocarditis team including surgical, infectious disease, and cardiology consultants.

The presence of high-degree AVB and clinical and echocardiographic features of cardiac amyloidosis suggest the utility of technetium-99m pyrophosphate for sensitive and specific for the non-biopsy detection of transthyretin cardiac amyloidosis.

\section{DISCRIMINATE INFLAMMATORY AORTOPATHIES FROM ACUTE AORTIC SYNDROME IN SELECTED CASES}

The association of intense FDG uptake in the aorta associated with elevations of CRP with CTA evidence of aortic wall thickening and presence of negative blood cultures for bacteria or fungus is strongly suggestive of aortitis. Further investigation is needed to clarify the significance of frequent findings of fasting FDG uptake in the ascending, arch, and descending aorta on routine fasting CS scans alone or in combination with classical findings of CS.

\section{ADVANTAGES OF NUCLEAR CARDIOLOGY IMAGING IN THE CICU}

Minimal patient cooperation is required. Imaging times are brief. CICU patients can be imaged with instrumentation and devices such as pacemakers, ICD, and balloon pumps. 
Imaging is not precluded by kidney or liver failure; vasodilator stress testing can be routinely performed in moderate-to-severe COPD and mild-to-moderate asthma.

In summary, the greatest values of nuclear cardiology include safety, effectiveness, and incremental value of diagnosis, prognosis, and assessment of treatment response. CICU patients can benefit greatly from nuclear cardiology, and diagnostic SPECT and PET imaging can be safely and effectively performed in patients with multi-organ failure and in the presence of cardiac devices such as pacemakers, ICD, or intra-aortic balloons. Advances in digital PET and CZT SPECT instrumentation provide similar spatial resolution, safe and effective low-dose imaging and rapid imaging results. This overview by our colleagues at the Cleveland Clinic reminds us that clinicians must understand current and future nuclear cardiology applications to optimize safety and outcomes for these acutely ill patients in the CICU who confront risks of imminent worsening of cardiovascular morbidity and mortality with downstream testing and interventions.

\section{References}

1. Dilsizian V, Gewirtz H, Paivanas N, Kitsiou AN, Hage FG, Crone $\mathrm{NE}$, et al. Serious and potentially life threatening complications of cardiac stress testing: Physiological mechanisms and management strategies. J Nucl Cardiol 2015;22:1198-213. https://doi.org/10. 1007/s12350-015-0141-4.

2. Doran JA, Sajjad W, Schneider MD, Gupta R, Mackin ML, Schwartz RG. Aminophylline and caffeine for reversal of adverse symptoms associated with regadenoson SPECT MPI. J Nucl Cardiol. 2017;24:1062-70. https://doi.org/10.1007/s12350-016-04 52-0.

3. Schenone AL, Hutt E, Cremer P et al. Utility of nuclear cardiovascular imaging in the cardiac intensive care unit. J. Nucl. Cardiol. (2021). https://doi.org/10.1007/s12350-021-02665-Z

4. Hachamovitch R, Hayes SW, Friedman JD, Cohen I, Berman DS. Comparison of the short-term survival benefit associated with revascularization compared with medical therapy in patients with no prior coronary artery disease undergoing stress myocardial perfusion single photon emission computed tomography. Circulation 2003;107:2900-7.

5. Hachamovitch R, Rozanski A, Shaw LJ, Stone GW, Thomson LE, Friedman JD, et al. Impact of ischaemia and scar on the therapeutic benefit derived from myocardial revascularization vs. medical therapy among patients undergoing stress-rest myocardial perfusion scintigraphy. Eur Heart J 2011;32:1012-24.

6. Taqueti VR, Hachamovitch R, Murthy VL, Naya M, Foster CR, Hainer $\mathbf{J}$, et al. Global coronary flow reserve is associated with adverse cardiovascular events independently of luminal angiographic severity and modifies the effect of early revascularization. Circulation 2015;131:19-27.

7. Gupta A, Taqueti VR, van de Hoef TP, Bajaj NS, Bravo PE, Murthy VL, et al. Integrated noninvasive physiological assessment of coronary circulatory function and impact on cardiovascular mortality in patients with stable coronary artery disease. Circulation 2017;136:2325-36.

8. Patel KK, Spertus JA, Chan PS, Sperry BW, Thompson RC, Al Badarin $\mathrm{F}$, et al. Extent of myocardial ischemia on positron emission tomography and survival benefit with early revascularization. J Am Coll Cardiol 2019;74:1645-54. https://doi.org/10. 1016/j.jacc.2019.07.055.

9. Patel KK, Spertus JA, Chan PS, Sperry BW, Thompson RC, Al Badarin F, et al. Myocardial blood flow reserve assessed by positron emission tomography myocardial perfusion imaging identifies patients with a survival benefit from early revascularization. Eur Heart J 2020;41:759-68.

10. Agostini D, Roule V, Nganoa C, Roth N, Baavour R, Parienti JJ, et al. First validation of myocardial flow reserve assessed by dynamic ${ }^{99 \mathrm{~m}} \mathrm{Tc}$-sestamibi CZT-SPECT camera: head to head comparison with ${ }^{15} \mathrm{O}$-water PET and fractional flow reserve in patients with suspected coronary artery disease. The WATERDAY study. Eur J Nucl Med Mol Imaging 2018;45:1079-90. https://doi. org/10.1007/s00259-018-3958-7.

11. De Souza ACDAH, Gonçalves BKD, Tedeschi AL, Lima RSL. Quantification of myocardial flow reserve using a gamma camera with solid-state cadmium-zinc-telluride detectors: Relation to angiographic coronary artery disease. J Nucl Cardiol 2019. http s://doi.org/10.1007/s12350-019-01775-z.

12. D'Egidio G, Nichol G, Williams KA, Guo A, Garrard L, deKemp $\mathrm{R}$, et al. Increasing benefit from revascularization is associated with increasing amounts of hibernating myocardium: A substudy of the PARR-2 trial. JACC Cardiovasc Imaging 2009;2:1060-8. h ttps://doi.org/10.1016/j.jcmg.2009.02.017.

13. Galassi AR, Centamore G, Fiscella A, Coppola A, Liberti F, Franco M, et al. Comparison of rest-redistribution thallium-201 imaging and reinjection after stress-redistribution for the assessment of myocardial viability in patients with left ventricular dysfunction secondary to coronary artery disease. Am J Cardiol 1995;75:436-42. https://doi.org/10.1016/s0002-9149(99)80577-3.

14. Schwartz RG, Malhotra S. Optimizing cardiac sarcoid imaging with FDG PET: Lessons from studies of physiologic regulation of myocardial fuel substrate utilization. J. Nucl. Cardiol 2020;27:490-3. https://doi.org/10.1007/s12350-018-1438-x.

15. Swart LE, Scholtens AM, Tanis W, Nieman K, Bogers AJ, Verzijlbergen FJ, et al. 18F-fluorodeoxyglucose positron emission/computed tomography 44 and computed tomography angiography in prosthetic heart valve endocarditis: From guidelines to clinical 45 practice. Eur Heart J 2018;39:3739-49. https://d oi.org/10.1093/eurheartj/ehx784.

16. Hyafil F, Rouzet F, Le Guludec D. Nuclear imaging for patients with a suspicion of infective endocarditis: Be part of the team! J Nucl Cardiol 2017;24:207-11. https://doi.org/10.1007/s12350-01 5-0369-z.

17. Roque A, Pizzi MN, Fernández-Hidalgo N, Permanyer E, CuellarCalabria H, Romero-Farina G, et al. Morpho-metabolic post-surgical patterns of non-infected prosthetic heart valves by [18F]FDG PET/CTA: "normality" is a possible diagnosis. Eur Heart J Cardiovasc Imaging 2020. https://doi.org/10.1093/ehjci/jez222.

18. Habib G, Lancellotti P, Antunes MJ, Bongiorni MG, Casalta J-P, Del Zotti F, et al. 2015 ESC Guidelines for the management of infective endocarditis. Eur Heart J 2015;36:3075-123. https://doi. org/10.1093/eurheartj/ehv319.

Publisher's Note Springer Nature remains neutral with regard to jurisdictional claims in published maps and institutional affiliations. 